\title{
Aging, physical activity, and diabetic complications related to loss of muscle strength in patients with type 2 diabetes
}

\author{
Takuo Nomura, PT, PhD, $\mathrm{CDEJ}^{1}$, Toshihiro KawaE, PT, MA, CDEJ ${ }^{2}$, \\ Hiroaki KATAOKA PT, PhD, $\mathrm{CDEJ}^{3}$ and Yukio IKEDA, MD, $\mathrm{PhD}^{4}$ \\ ${ }^{1)}$ Department of Rehabilitation Sciences, Kansai University of Welfare Sciences \\ ${ }^{2)}$ Division of Rehabilitation, Hiroshima University Hospital \\ ${ }^{3)}$ Rehabilitation Center, KKR Takamatsu Hospital \\ ${ }^{4)}$ Diabetes Center, Kochi Memorial Hospital
}

\begin{abstract}
Patients with type 2 diabetes may have motor dysfunctions such as loss of muscle strength. Compared with non-diabetic subjects, patients with diabetes show decreased lower extremity muscle strength. The aim of this review was to describe the influence of factors associated with loss of muscle strength in patients with type 2 diabetes. Aging promotes an accelerated loss of muscle strength in patients with diabetes. Physical inactivity may cause a decline in muscle strength in patients with diabetes. Gradual loss of muscle strength is related to the presence and severity of diabetic neuropathy. Diabetic nephropathy may be a factor contributing to loss of muscle strength, because decrease in skeletal muscle mass is a hallmark of end-stage renal disease. Resistance exercise is an essential component of diabetes treatment regimens and also plays a role in the prevention and management of sarcopenia. Intensive physical therapy intervention should be provided to patients with diabetes having decreased muscle strength.

Key words: type 2 diabetes, muscle strength, aging, physical activity, diabetic complications
\end{abstract}

(Phys Ther Res 21: 33-38, 2018)

The prevalence of type 2 diabetes is increasing worldwide $^{1)}$. According to a previous study, diabetic patients may present with motor skills deficits ${ }^{2}$; additionally, an early decline in physical function with age has been associated with type 2 diabetes $^{3)}$. These facts represent a serious problem, as the number of people aged $>65$ years is expected to continue to rise in developed countries such as Japan, Germany, and Italy ${ }^{4}$. The discovery of more effective measures to prevent movement disorders ${ }^{5)}$ is essential for patients with diabetes in a country with an increasingly aging population. In the elderly, mobility-related fatigue is associated with slower walking speed, and current evidence suggests that muscle strength is one of the underlying factors ex-

Received: July 9, 2018

Accepted: August 27, 2018

Advance Publication by J-STAGE: November 30, 2018

Correspondence to: Takuo Nomura, Department of Rehabilitation Sciences, Kansai University of Welfare Sciences, 3-11-1 Asahigaoka, Kashiwara city, Osaka 582-0026, Japan

\# e-mail: nomurata1017@yahoo.co.jp and

tnomura@tamateyama.ac.jp

doi: 10.1298/ptr.R0002 plaining this association ${ }^{6)}$. Furthermore, in elderly patients, leg muscle strength has been shown to be associated with balance and mobility skills ${ }^{7)}$. Therefore, it is important that healthcare professionals and medical teams pay attention to the loss of muscle strength in patients with diabetes.

In the present review article, we describe the influence of factors associated with loss of muscle strength in patients with type 2 diabetes.

\section{Muscle Strength in Diabetes}

Insulin resistance, hyperglycemia, muscle fat infiltration, peripheral neuropathies, and oxidative stress are hypothesized as the fundamental biological mechanisms leading to impairments in muscle strength in people with diabetes ${ }^{8,9)}$. Our previous study suggested that knee extension force (KEF) is independently associated with insulin resistance in patients with type 2 diabetes ${ }^{10)}$ (Table 1 ). These factors are likely to affect muscle strength in patients with type 2 diabetes.

We examined reference values for KEF (kgf; absolute value) and \% $\mathrm{KEF}$ (\%; body weight ratio) based on data 


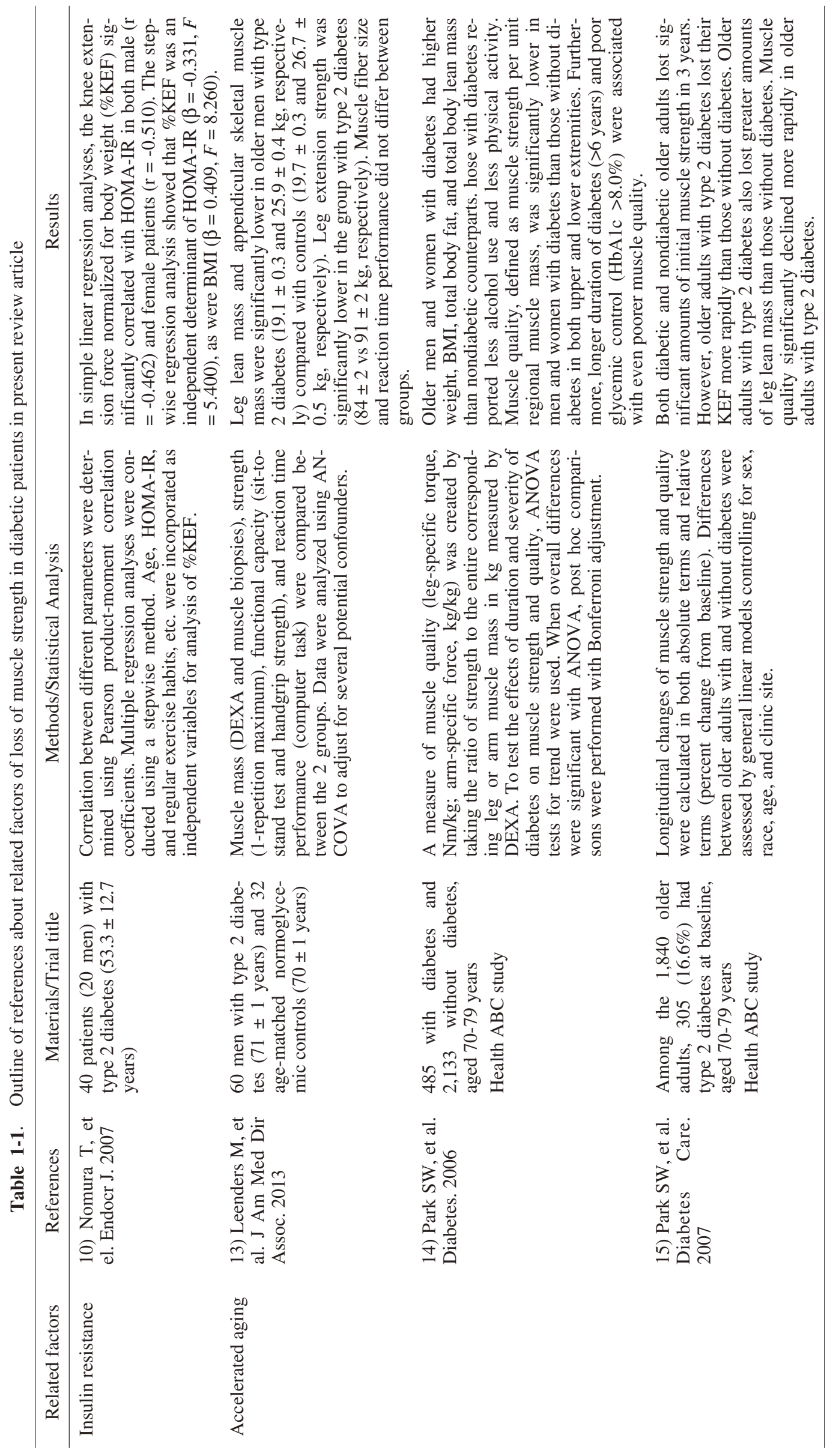




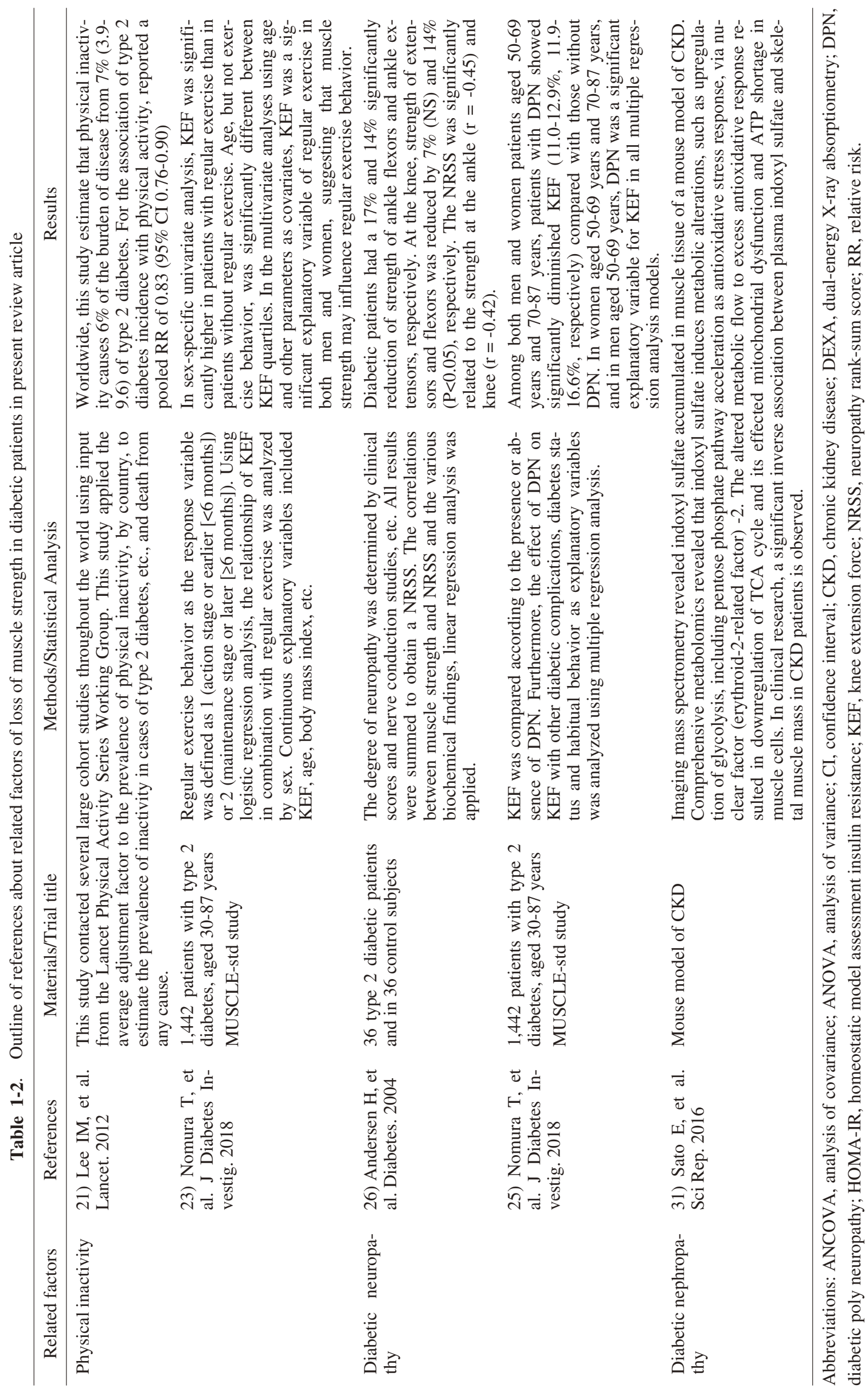


from type 2 diabetic patients with a wide age range who did not have diabetic polyneuropathy (DPN). In type 2 diabetic patients without apparent DPN compared with non-diabetic subjects, KEF and \% KEF may be reduced by approximately $10 \%$ and $20 \%$, respectively ${ }^{11)}$.

\section{Aging Muscle and Relation to Diabetes}

There is an interrelationship between muscle strength and aging ${ }^{12)}$. A natural decrease in muscle strength occurs with increasing age; however, patients with type 2 diabetes show greater decline in muscle strength with age ${ }^{13,14}$. Furthermore, accelerated loss of muscle strength is observed in elderly patients with type 2 diabetes ${ }^{15)}$. Loss of muscle strength is a predictor of functional limitations ${ }^{16)}$; muscle strength is also the single best measure of age-related muscle change and is associated with physical disability in instrumental activities of daily living. Moreover, diabetesrelated loss of muscle strength predisposes this population to a higher fall risk $^{17,18)}$.

\section{Regular Physical Activity and Physical Function in Patients with Diabetes}

Physical activity (PA) has been associated with better mobility in the elderly ${ }^{19,20)}$. The associations between physical inactivity and type 2 diabetes is well known ${ }^{21}$. The relationship between PA and physical function (PF) is bidirectional, with PF more consistently predicting declines of $\mathrm{PA}^{22)}$. In a sex-specific univariate analysis, KEF was significantly higher in patients who regularly exercised than in patients who did not regularly exercise ${ }^{23}$. Moreover, in the multivariate analyses using age and other parameters as covariates, KEF was found to be a significant explanatory variable of regular exercise in both men and women, suggesting that muscle strength could influence regular exercise behavior.

\section{Loss of Muscle Strength Related to Diabetic Neuropathy}

Demyelination and axonal degeneration are established hallmarks of diabetic neuropathy (DN) pathophysiol$\mathrm{ogy}^{24}$. One of the common forms of DN is DPN, which is further classified into a sensory nerve disorder, motor nerve disorder, or autonomic nerve disorder. DPN is a diabetic complication to most clinically combine to patients with diabetes. In our previous study, the incidence of DPN was $37.7 \%$ in 1,442 patients with type 2 diabetes ${ }^{25}$.

The gradual loss of muscle strength in type 2 diabetes is related to the presence and severity of $\mathrm{DPN}^{26)}$. No population-based studies on this topic have been carried out, and the characteristics of diminished muscle strength according to sex or age group have not been determined. In our previous study, comparisons of KEF according to sex and age group showed neither men nor women aged 30-49 years had a significant difference in KEF based on their DPN status. On the other hand, both men and women participants aged 50-69 years and 70-87 years with DPN showed a significantly diminished KEF by $10.9-16.5 \%$ compared with those without $\mathrm{DPN}^{25)}$. These results show that DPN might accelerate lower extremity muscle strength decline in middle-aged and elderly type 2 patients with diabetes.

\section{Loss of Muscle Strength Related to Diabetic Nephropathy}

Diabetes affects the small blood vessels in the glomerulus, a key structure in the kidney composed of capillary blood vessels. Diabetic nephropathy represents a leading cause of ongoing dialysis ${ }^{27)}$, accounting for $38.4 \%$ of dialysis usage in Japan in $2015^{28)}$.

Chronic kidney disease (CKD) as a clinical entity is a relatively new concept, with diabetes and high blood pressure being listed as the two main causes ${ }^{29)}$. It is known that elderly patients with CKD show decreased physical function, quality of life, and mental health ${ }^{30}$. Sato et al. reported that the causative pathological mechanism of uremic sarcopenia is metabolic alterations by uremic toxin indoxyl sulfate in a mouse model of $\mathrm{CKD}^{31}$. Moreover, a significant inverse association between plasma indoxyl sulfate and skeletal muscle mass in CKD patients was observed in clinical research ${ }^{31)}$. Previous reports indicate that indoxyl sulfate may be a pathogenic factor for sarcopenia in CKD. These facts show that skeletal muscle dysfunction and poor exercise tolerance are hallmarks of end-stage renal disease.

\section{Exercise for Loss of Muscle Strength in Patients with Diabetes}

Exercise therapy is well-established as a fundamental treatment for patients with type 2 diabetes ${ }^{32}$. Two possible exercise interventions for type 2 diabetes are aerobic and resistance exercise; more specifically, moderate- or highintensity resistance exercise has been reported as a potentially effective means to improve muscle strength and physical function ${ }^{33)}$. Resistance exercise is a crucial means to treat diabetes-related loss of muscle strength and prevent and manage sarcopenia. However, strict blood pressure control is required according to the severity of the diabetic complications such as diabetic retinopathy and diabetic nephropathy ${ }^{34)}$. High-intensity exercise therapy raises blood pressure; therefore, it is essential to make the appropriate individual adjustments. 


\section{Conclusion}

This review discussed factors related to the loss of muscle strength in patients with type 2 diabetes. There is an acceleration in the typical age-related decrease in muscle strength in elderly patients with diabetes. Current literature shows that the presence of DPN is the main factor contributing to loss of muscle strength in patients with type 2 diabetes; this fact is especially clear in middle-aged and elderly patients. Skeletal muscle dysfunction and poor exercise tolerance are hallmarks of end-stage renal disease. Intensive physical therapy intervention should be provided to diabetic patients with a loss of muscle strength ${ }^{35}$.

Funding: This work was supported by the Japan Society for the Promotion of Science (JSPS) KAKENHI Grant Numbers JP15K01440. Publication of this paper received a research grant from the Kansai University of Welfare Sciences.

Conflict of Interest: Authors have no conflict of interests to disclose.

\section{References}

1) International Diabetes Federation: Diabetes Atlas, 8 th edition. 2017: [cited 2018 May 30]; Available from: http://www.diabetes atlas.org/resources/2017-atlas.html.

2) Andersen H: Motor dysfunction in diabetes. Diabetes Metab Res Rev. 2012; 28(Suppl 1): 89-92.

3) Fritschi C, Bronas UG, et al.: Early declines in physical function among aging adults with type 2 diabetes. J Diabetes Complications. 2017; 31: 347-352.

4) Organisation for Economic Cooperation and Development: Elderly population. [cited 2018 May 30]; Available from: https://dat a.oecd.org/pop/elderly-population.htm.

5) Yamada K, Muranaga S, et al.: Age independency of mobility decrease assessed using the Locomotive Syndrome Risk Test in elderly with disability: a cross-sectional study. BMC Geriatr. 2018; 18: 28.

6) Mänty M, de Leon CF, et al.: Mobility-related fatigue, walking speed, and muscle strength in older people. J Gerontol A Biol Sci Med Sci. 2012; 67: 523-529.

7) Hasselgren L, Olsson LL, et al.: Is leg muscle strength correlated with functional balance and mobility among inpatients in geriatric rehabilitation? Arch Gerontol Geriatr. 2011; 52: e220225.

8) Bianchi L and Volpato S: Muscle dysfunction in type 2 diabetes: a major threat to patient's mobility and independence. Acta Diabetol. 2016; 53: 879-889.

9) Tsutsui H, Kinugawa S, et al.: Oxidative stress in cardiac and skeletal muscle dysfunction associated with diabetes mellitus. J Clin Biochem Nutr. 2011; 48: 68-71.

10) Nomura $T$, Ikeda $Y$, et al:: Muscle strength is a marker of insulin resistance in patients with type 2 diabetes: a pilot study. Endocr
J. 2007; 54: 791-796.

11) Nomura T: A meaning as view from the epidemiology for muscle volume and muscle strength in patients with diabetes. In: Tamura Y (ed): Exercise Therapy in Diabetes: Science \& Practice. 1 st ed, Ishiyaku Publisher Inc, Tokyo, 2018, pp. 20-26(Japanese).

12) Kaya RD, Nakazawa M, et al.: Interrelationship between muscle strength, motor units, and aging. Exp Gerontol. 2013; 48: 920925.

13) Leenders M, Verdijk LB, et al.: Patients with type 2 diabetes show a greater decline in muscle mass, muscle strength, and functional capacity with aging. J Am Med Dir Assoc. 2013; 14: 585-592.

14) Park SW, Goodpaster BH, et al.: Decreased muscle strength and quality in older adults with type 2 diabetes: the health, aging, and body composition study. Diabetes. 2006; 55: 1813-1818.

15) Park SW, Goodpaster BH, et al.: Accelerated loss of skeletal muscle strength in older adults with type 2 diabetes: the health, aging, and body composition study. Diabetes Care. 2007; 30: 1507-1512.

16) Hairi NN, Cumming RG, et al.: Loss of muscle strength, mass (sarcopenia), and quality (specific force) and its relationship with functional limitation and physical disability: the Concord Health and Ageing in Men Project. J Am Geriatr Soc. 2010; 58 : 2055-2062.

17) Crews RT, Yalla SV, et al.: A growing troubling triad: diabetes, aging, and falls. J Aging Res. 2013; 2013: 342650.

18) Vinik AI, Camacho P, et al.: Aging, diabetes, and falls. Endocr Pract. 2017; 23: 1117-1139.

19) Tikkanen P, Nykanen I, et al.: Physical activity at age of 20-64 years and mobility and muscle strength in old age: a communitybased study. J Gerontol A Biol Sci Med Sci. 2012; 67: 905-910.

20) Brach JS, Simonsick EM, et al.: The association between physical function and lifestyle activity and exercise in the health, aging and body composition study. J Am Geriatr Soc. 2004; 52: 502-509.

21) Lee IM, Shiroma EJ, et al:: Effect of physical inactivity on major non-communicable diseases worldwide: an analysis of burden of disease and life expectancy. Lancet. 2012; 380: 219-229.

22) Metti AL, Best JR, et al.: Longitudinal changes in physical function and physical activity in older adults. Age Ageing. [cited 2018 Mar. 13]; Available from: doi: 10.1093/ageing/afy025. [Ep ub ahead of print].

23) Nomura T, Ishiguro T, et al.: Regular exercise behavior is related to lower extremity muscle strength in patients with type 2 diabetes: Data from the Multicenter Survey of the Isometric Lower Extremity Strength in Type 2 Diabetes study. J Diabetes Investig. 2018; 9: 426-429.

24) Malik RA: Pathology of human diabetic neuropathy. Chap 18. In: Rochodne DW and Malik RA (eds): Handbook of Clinical Neurology, Elsevier B.V., Amsterdam, 2014, pp. 249-259.

25) Nomura $T$, Ishiguro $T$, et al.: Diabetic polyneuropathy is a risk factor for decline of lower extremity strength in patients with type 2 diabetes. J Diabetes Investig. 2018; 9: 186-192.

26) Andersen $\mathrm{H}$, Nielsen $\mathrm{S}$, et al.: Muscle strength in type 2 diabe- 
tes. Diabetes. 2004; 53: 1543-1548.

27) Sugiyama T, Goryoda S, et al.: Construction of a simulation model and evaluation of the effect of potential interventions on the incidence of diabetes and initiation of dialysis due to diabetic nephropathy in Japan. BMC Health Serv Res. 2017; 17: 833.

28) Masakane I, Taniguchi M, et al.: Annual Dialysis Data Report 2015, JSDT Renal Data Registry. Renal Replacement Therapy. 2018; 4: Available from: https://doi.org/10.1186/s41100-018-01 49-8.

29) Inker LA, Astor BC, et al.: KDOQI US commentary on the 2012 KDIGO clinical practice guideline for the evaluation and management of CKD. Am J Kidney Dis. 2014; 63: 713-735.

30) Martini A, Ammirati A, et al.: Evaluation of quality of life, physical, and mental aspects in longevous patients with chronic kidney disease. Int Urol Nephrol. 2018; 50: 725-731.

31) Sato E, Mori T, et al.: Metabolic alterations by indoxyl sulfate in skeletal muscle induce uremic sarcopenia in chronic kidney dis- ease. Sci Rep. 2016; 6: 36618.

32) Colberg SR, Sigal RJ, et al.: Physical activity/exercise and diabetes: A position statement of the American Diabetes Association. Diabetes Care. 2016; 39: 2065-2079.

33) Cruz-Jentoft AJ, Landi F, et al.: Prevalence of and interventions for sarcopenia in ageing adults: a systematic review. Report of the International Sarcopenia Initiative (EWGSOP and IWGS). Age Ageing. 2014; 43: 748-759.

34) Chamberlain JJ, Rhinehart AS, et al.: Diagnosis and management of diabetes: Synopsis of the 2016 American Diabetes Association Standards of Medical Care in Diabetes. Ann Intern Med. 2016; 164: 542-552.

35) Nomura T, Kawae T, et al:: Assessment of lower extremity muscle mass, muscle strength, and exercise therapy in elderly patients with diabetes mellitus. Environ Health Prev Med. 2018; 23: 20. [cited 2018 May 17]; Available from: doi: 10.1186/s121 99-018-0710-7. 